\title{
The influences of reproductive status and acute stress on the levels of phosphorylated delta opioid receptor immunoreactivity in rat hippocampus
}

\author{
Suzanne R. Burstein ${ }^{\mathrm{a}}$, Tanya J. Williams ${ }^{\mathrm{a}, \mathrm{b}}$, Diane A. Lane ${ }^{\mathrm{a}}$, Margarete G. Knudsen ${ }^{\mathrm{a}}$, \\ Virginia M. Pickel ${ }^{a}$, Bruce S. McEwen ${ }^{c}$, Elizabeth M. Waters ${ }^{c}$, and Teresa A. Milner ${ }^{a, c,}{ }^{*}$ \\ Teresa A. Milner: tmilner@med.cornell.edu \\ aBrain and Mind Research Institute, Weill Cornell Medical College, 407 East 61st Street, New \\ York, NY 10065, USA \\ bWeill Cornell/Rockefeller/Sloan-Kettering Tri-Institutional MD-PhD Program, New York, NY \\ 10065, USA \\ cHarold and Margaret Milliken Hatch Laboratory of Neuroendocrinology, The Rockefeller \\ University, 1230 York Avenue, New York, NY 10065, USA
}

\begin{abstract}
In the hippocampus, ovarian hormones and sex can alter the trafficking of delta opioid receptors (DORs) and the proportion of DORs that colocalize with the stress hormone, corticotropin releasing factor. Here, we assessed the effects of acute immobilization stress (AIS) and sex on the phosphorylation of DORs in the rat hippocampus. We first localized an antibody to phosphorylated DOR (pDOR) at the SER363 carboxy-terminal residue, and demonstrated its response to an opioid agonist. By light microscopy, pDOR-immunoreactivity (ir) was located predominantly in CA2/CA3a pyramidal cell apical dendrites and in interneurons in CA1-3 stratum oriens and the dentate hilus. By electron microscopy, pDOR-ir primarily was located in somata and dendrites, associated with endomembranes, or in dendritic spines. pDOR-ir was less frequently found in mossy fibers terminals. Quantitative light microscopy revealed a significant increase in pDOR-ir in the CA2/CA3a region of male rats $1 \mathrm{~h}$ following an injection of the opioid agonist morphine $(20 \mathrm{mg} / \mathrm{kg}$, I.P). To look at the effects of stress on pDOR, we compared pDORir in males and cycling females after AIS. The level of pDOR-ir in stratum radiatum of CA2/CA3a was increased in control estrus (elevated estrogen and progesterone) females compared to proestrus and diestrus females and males. However, immediately following $30 \mathrm{~min}$ of AIS, no significant differences in pDOR levels were seen across estrous cycle phase or sex. These findings suggest that hippocampal levels of phosphorylated DORs vary with estrous cycle phase and that acute stress may dampen the differential effects of hormones on DOR activation in females.
\end{abstract}

\section{Keywords}

Opioids; Sex differences; Estrogens; CA2 region of the hippocampus; Pyramidal cells; Mossy fiber pathway

\footnotetext{
* Grant support: NIH grants DA08259 \& HL098351 (TAM), HL096571 (TAM and VMP), T32 DA007274 (DL), MSTP grant GM07739 (TJW), DA004600 (VMP), DK07313 \& AG039850 (EMW), NS007080 (BSM), DA028072 (TJW), the UNCF-Merck Science Initiative (TJW).

(C) 2013 Elsevier B.V. All rights reserved.

"Corresponding author at: Brain and Mind Research Institute, Weill Cornell Medical College, 407 East 61st Street, RM 307, New York, NY 10065, USA. Fax: +1 6469620535.
} 


\section{Introduction}

Opiate addiction involves both drug-related reward circuitry and spatial/episodic memory (Koob et al., 1998; Hyman and Malenka, 2001; Nestler, 2002). Stress has long been known to be a contributing factor in drug abuse, however men and women respond differently. For instance, women are more prone to relapse in a stress-provoking situation than men (Rubin et al., 1996; McKay et al., 1996; Elman et al., 2001; Koob, 2008).

Of particular relevance to the addictive processes are sexually dimorphic effects of stress on hippocampal-related learning and circuitry. In males, chronic stress impairs cognitive performance on spatial memory tasks as well as non-spatial recognition memory tasks and decreases long-term potentiation (for reviews see (Luine et al., 2007; McEwen and Milner, 2007)). Moreover, chronic stress in males results in atrophy and debranching of CA3 pyramidal cell apical dendrites (McEwen, 1999; Vyas et al., 2002), increases the packing density of small synaptic vesicles near active zones in mossy fiber terminals (Magarinos et al., 1997) and decreases the number of interneurons containing parvalbumin (Hu et al., 2010; Czeh et al., 2005). Conversely, chronic stress in females enhances performance on the same memory tasks (Conrad et al., 2003; Kitraki et al., 2004; Luine et al., 2007) and does not result in severe atrophy of CA3 pyramidal cell apical dendrites (Galea et al., 1997) or the loss of parvalbumin-containing interneurons (Milner unpublished).

Hippocampal regions vulnerable to sexually dimorphic effects of stress extensively overlap with the endogenous opioid system, a system that is important in drug-related learning [reviewed in Drake et al. (2007)]. In particular, enkephalins and dynorphins are contained in the mossy fiber pathway, whereas delta and mu opioid receptors (DORs and MORs) are expressed in pyramidal cells and parvalbumin interneurons, respectively (Drake et al., 2007). Activation of MORs and DORs, either by endogenous opioid peptides or exogenous agonists such as morphine can increase pyramidal cell excitability and can facilitate longterm-potentiation [reviewed by Simmons and Chavkin (1996)].

Emerging evidence indicates that both ovarian hormones and stress affect the hippocampal opioid system. Opioid peptide levels in the mossy fiber pathway (Torres-Reveron et al., 2008, 2009a) as well as trafficking of MORs and DORs in hippocampal neurons (TorresReveron et al., 2009b; Williams et al., 2011b) are sensitive to ovarian hormone levels. DORcontaining neurons also express the stress hormone corticotropin releasing factor (CRF) and its receptor, and the proportion of co-labeling for these proteins varies with sex (Williams and Milner, 2011; Williams et al., 2011a). Moreover, both acute and chronic stress can alter the trafficking and levels of MORs in parvalbumin-containing interneurons and the activation of MORs in a sex-dependent manner (Gonzales et al., 2011; Milner et al., 2011a; Khalid et al., 2010). However, whether stress affects hippocampal DORs is not known.

Thus, the present study sought to determine if DORs also are phosphorylated in the hippocampus following stress, and if it differs in males and cycling females. To do so, we used an antibody to the phosphorylated delta opioid receptor (pDOR) to examine the localization of pDOR via light and electron microscopy in males and cycling females after acute immobilization stress (AIS).

\section{Results}

\section{1. pDOR-ir is found in select subregions the hippocampus}

To determine the cellular distribution of phosphorylated DORs, pDOR labeling was examined by light microscopy using peroxidase immunocytochemistry. pDOR-

immunoreactivity (ir) was limited to select subregions in the rat hippocampus, particularly to 
CA2/CA3a and CA1-FC (Fig. 1). pDOR-ir was found primarily in CA2/CA3a pyramidal cell bodies and their apical dendrites in stratum radiatum (Fig. 1A and B). Moreover, pDORir was found in scattered interneurons in the dorsal and central portions of the hilus of the dentate gyrus (Fig. 1C) and throughout stratum oriens of CA1-CA3. Neurons containing pDOR-ir also were seen in the fasciola cinera, where it borders the most rostral portion of CA1 (Fig. 1D). The pattern of pDOR-labeling appeared identical in male and female rats.

\section{2. pDOR-ir is primarily in somata and dendrites}

To determine the subcellular localization of pDOR-ir, regions of the hippocampus showing the most dense labeling by light microscopy were analyzed using electron microscopy. Ultrastructural analysis of the CA2/CA3a region revealed that pDOR-ir was found exclusively in the somata and dendrites of pyramidal cells (Figs. 2 and 3). Pyramidal cell somata were identified by their large size $(20-25 \mu \mathrm{m}$ in diameter) and their presence in the pyramidal cell layer. Proximal dendrites that emanated from pyramidal cell somata were often contacted by large mossy fiber boutons (Fig. 3A). More distal pyramidal cell dendrites were identified by the presence of spines (Fig. 3B and C). In soma and large proximal dendrites, patches of pDOR-ir were localized to endoplasmic reticula (Fig. 2). In more distal dendrites, pDOR-ir was associated with endomembranes (Fig. 3A and C). Occasionally, pDOR-ir was also found on the plasma membrane of distal dendrites (Fig. 3B).

In the hilus of the dentate gyrus, electron microscopic analysis revealed that pDOR-ir was exclusively found in neuronal profiles. Most of the pDOR-labeled profiles were dendrites although some pDOR-labeled terminals also were found (Fig. 4). Like CA2/CA3, pDOR-ir in the hilus was punctate and associated with endomembranes (not shown). Moreover, dense pDOR-ir was seen in dendritic spine heads (Fig. 4A and B). pDOR-labeled spines were contacted by unlabeled terminals that formed both monosynaptic (Fig. 4A) and disynaptic (Fig. 4B) synapses. Less frequently, pDOR-ir was detected in mossy fiber terminals (Fig. 4C). Within terminals, pDOR-ir was usually patchy and affiliated with vesicles. Most pDOR-labeled terminals formed asymmetric synapses on unlabeled dendritic spines (Fig. 4C).

\section{3. pDOR levels in CA2/CA3 increase following morphine administration}

We next determined if an opioid agonist, morphine, could increase pDOR-ir in male rats. For this, quantitative light microscopic immunocytochemistry was used to analyze hippocampal sections from male rats injected with morphine $(20 \mathrm{mg} / \mathrm{kg}$, I.P.) or saline and sacrificed $1 \mathrm{~h}$ later (Lane et al., 2008). The level of pDOR-ir was examined in four hippocampal subregions Fig. 5; in each subregionx measurements were taken over the regions containing cell bodies and proximal apical dendrites. Morphine administration produced a significant increase in pDOR-ir $(F(1,106)=21.08, p<0.0001)$. There was also a significant difference in $\mathrm{pDOR}$-ir by the hippocampal region $(F(3,106)=266.02, p=0.0001)$. There was no interaction effect between drug administration and hippocampal region $(F(3,138)=1.50, p>40.05)$ because morphine increased pDOR-ir in all regions of the hippocampus, although it was more pronounced in CA1-FC and CA2/CA3a.

\subsection{Alterations in pDOR-ir following AIS vary depending on region and hormonal status}

We next determined if the levels of pDOR-ir differ in response to gonadal steroid status and following AIS. For this, pDOR-ir level in CA2/CA3a (Fig. 6) was quantified using light microscopic densitometry in males and females from each estrous cycle phase following AIS. There was a significant effect of estrous cycle on pDOR-ir $(F(3,37)=8.27, p=0.01)$. There was no difference in pDOR-ir with stress $(F(1,37)=1.05, p>0.05)$. There was, however, a significant interaction between estrous cycle and stress $(F(3,37)=4.15, p<0.05)$. 
To determine if estrogen contributed to the increased pDOR-ir seen in estrus females, quantitative densitometry was used to assess pDOR-ir in ovariectomized rats $24 \mathrm{~h}$ following estradiol benzoate $(10 \mu \mathrm{g} / 0.2 \mathrm{ml})$ administration. This time-point and dose of estradiol benzoate was chosen as previous studies have shown increased hippocampal mossy fiber enkephalin and dynorphin levels in response to this treatment (Torres-Reveron et al., 2008, 2009a). The density of pDOR-ir was examined in CA2/CA3a and the hilus following estradiol benzoate administration to ovariectomized rats (data not shown). There was no significant effect of estrogen treatment $(F(1,40)=3.70, p>0.05)$ or significant interaction effect between estrogen treatment and hippocampal region $(F(1,40)=0.07, p>0.05)$. There was a significant difference between hippocampal regions $(F(1,40)=868.40, p<0.0001)$, which is not surprising given the large difference in pDOR labeling between the hilus and CA1.

\section{Discussion}

This study demonstrates that pDOR-ir is localized to select subregions of the hippocampus where the density of labeling is increased in response to morphine. In contrast to phosphorylated MOR-ir (Gonzales et al., 2011), the levels of pDOR-ir are increased in unstressed female rats during estrus. However, the observed increase in pDOR-ir level in female rats is no longer apparent following acute stress. This suggests that acute stress induces signaling changes in the hippocampus that dampen the effect of high levels of estrogen on DOR activation.

\subsection{Methodological considerations}

We used a well-characterized antibody to pDOR (Ser363). In Western blots of mouse hippocampal samples collected 20 min following injection of DOR agonist SNC80 (10 mg/ $\mathrm{kg}$, I. P.) a $65 \mathrm{kDa}$ band was detected; in hippocampal samples from DOR knockout mice, similarly injected, no $65 \mathrm{kDa}$ band was detected (Pradhan et al., 2009). This is the first report to use the pDOR (Ser363) antiserum in acrolein/paraformaldehyde fixed neuronal tissue. This study also shows that morphine induces a significant increase in pDOR-ir in the areas where it is highly expressed, mainly in pyramidal cells in hippocampal subregions CA1-FC and CA2/CA3a.

We did not find an effect of estrogen treatment in ovariectomized animals on the levels of pDOR-ir in the hippocampus. In this study, pDOR-ir was examined at $24 \mathrm{~h}$ following estrogen replacement, when leu-enkephalin and dynorphin are elevated in the mossy fiber pathway (Torres-Reveron et al., 2008, 2009a). However, the levels of hippocampal pDOR-ir did not change at this time-point. Several of the most frequently used ovariectomy models vary by hormone replacement dose, time interval between ovariectomy and hormone replacement, and time interval between hormone replacement and analysis, and these differences can influence estrogen-mediated effects (Adams et al., 2001; Tanapat et al., 2005). Thus, it is possible that time-points and dosing regimens of estradiol not employed in the current study could affect pDOR-ir levels in the hippocampus.

\section{2. pDOR-ir is mostly in CA2/CA3a pyramidal cells and scattered hilar interneurons}

This study reveals that pDOR-ir is predominant in pyramidal cells in the CA2/CA3a region of the hippocampus. The localization of pDOR in pyramidal cells is consistent with mRNA and immunocytochemistry studies of DOR (Stumm et al., 2004; Williams et al., 2011b), however DOR localization is not as heavily concentrated in the CA2/CA3a region as pDOR. Recent studies have shown that CA2 pyramidal neurons are distinct in morphology and may have a different function than in CA1 and CA3 (Mercer et al., 2007; Jones and McHugh, 2011). CA2/CA3a neurons have an overlapping distribution with mossy fiber boutons, 
which contain a buildup of opioid peptides (Torres-Reveron et al., 2008; Pierce et al., 1999). CA2 is the only pyramidal cell region innervated by the supramammillary nucleus (Magloczky et al., 1994), a hypothalamic nucleus believed to participate in theta rhythm generation (Vertes and McKenna, 2000). The CA2 region is also notable because it highly expresses adenosine $A 1$ receptors $\left(\mathrm{A}_{1} \mathrm{Rs}\right)$, which have recently been shown to bind caffeine and act to induce long-lasting potentiation of synaptic transmission (Simons et al., 2011; Ochiishi et al., 1999). Moreover, activation of $\mathrm{A}_{1}$ Rs causes phosphorylation and desensitization of DORs, suggesting a functional interaction between these two receptors (Cheng et al., 2010).

pDOR-ir was also found in hilar interneurons consistent with previous studies that have localized DORs to somatostatin and neuropeptide $\mathrm{Y}$ containing interneurons in this brain region (, 1997; Williams et al., 2011b). In interneurons, DOR activation indirectly excites pyramidal cells (Stumm et al., 2004). Moreover, DORs have been localized to CRFcontaining interneurons, likely hilar perforant path (HIPP) associated cells, in the dentate hilus (Williams et al., 2011a), an interneuron subgroup that regulates granule cell input and LTP at the perforant path—granule cell synapse as shown by extensive work characterizing interneurons in the hippocampus (reviewed by Freund and Buzsaki (1996)). As both DOR and CRF have been shown to modulate LTP (Bramham et al., 1991; Wang et al., 1998), these $\mathrm{pDOR}$ - containing neurons are positioned to modulate hippocampal signaling that is involved in the stress response.

High levels of pDOR-ir were also detected in the fasciola cinera, which is part of the supracallosal gyrus and continuous with the dentate gyrus (Amaral and Lavenex, 2007). The neuropeptide Y receptor Y5 also displays the most immunoreactive staining in both the $\mathrm{CA} 2 / \mathrm{CA} 3 \mathrm{a}$ and fasciola cinera regions in mainly pyramidal cells and some hilar interneurons (Grove et al., 2000). Interestingly, CA3 NPY-labeled interneurons contain fewer DORs in proestrus females compared to males (Williams et al., 2011b). Whether a similar relationship exists in the fasciola cinera is unknown. However, this suggests that interactions between endogenous opioids and neuropeptide $\mathrm{Y}$ may be influenced by gonadal steroids.

\section{3. pDOR-ir is positioned to regulate synaptic and non-synaptic transmission}

Electron microscopy revealed that pDOR-ir in the hippocampus is mostly in somata and dendrites. The localization of pDOR-ir near endomembranes and the endoplasmic reticulum is consistent with involvement of these organelles in the synthesis and trafficking of receptor proteins. When an agonist binds to a $\mathrm{G}$ protein coupled receptor, in addition to activating a signal cascade, it is also phosphorylated by GPCR kinases and internalized as part of a feedback regulatory process. For many receptors, including DORs, once the ligand binds, receptor phosphorylation parallels uncoupling and internalization of the receptor (Pradhan et al., 2009). Removal of the receptor from the plasma membrane surface can lead to desensitization and may contribute to tolerance effects (Koch and Hollt, 2008; Scherrer et al., 2006; Wang and Wang, 2006). pDOR-ir also is found near the plasma membrane and at postsynaptic densities in dendritic spines. At this location, activated DORs could affect local synaptic signaling. In particular, DOR selective ligands can inhibit intracellular cAMP levels and modulate the activity of voltage-gated calcium and potassium channels as well as protein kinases including PKC and MAPK (Quock et al., 1999). Recently, we have shown that DORs are colocalized with CRF receptors in hippocampal neurons, ideally positioning them to modulate CRF receptor signaling (Williams et al., 2011a).

DORs in CA2/3 pyramidal cells and hilar interneurons could be activated by either exogenous or endogenous opioids. The former assertion is supported by our finding that pDOR-ir is elevated in CA2/3 $1 \mathrm{~h}$ following morphine administration. Endogenous opioids 
are stored in dense-core vesicles within mossy fiber terminals (Commons and Milner, 1995; Pierce et al., 1999). Previous studies have shown that swim stress or seizure can release opioid peptides in the hippocampus (Pierce et al., 1999; Redila and Chavkin, 2008). Thus, it is likely that immobilization stress similarly can release opioids from dense core vesicles.

A minority of pDOR-ir was found in axon terminals, some of which were mossy fibers. In presynaptic terminals in rat hippocampal interneurons, DOR activation inhibits GABA release (Cohen et al., 1992). Within terminals, pDOR-ir was mostly associated with clusters of small synaptic vesicles. Since these small synaptic terminals contain glutamate or GABA, the present findings suggest that DOR activation may affect transmission of these transmitters.

\subsection{Opioid receptors and acute stress}

While pDOR-ir is elevated in unstressed female rats in estrus, following AIS this elevation is lost. This finding contrasts those of pMOR following AIS (Gonzales et al., 2011). In the hippocampus, pMOR-ir is predominantly found presynaptically in mossy fiber boutons and axons and in a select population of hilar interneurons (Gonzales et al., 2011). The levels of pMOR-ir are similar in normal unstressed male rats and female rats and do no vary across the estrous cycle. However, sex and cycle differences emerged following AIS in a hippocampal region-specific manner: pMOR-ir increased in male rats and decreased in proestrus (high estrogen) females in the dentate hilus (Gonzales et al., 2011). Given that pMOR is largely presynaptic, and pDOR is mostly postsynaptic, the activated forms of the MOR and DOR may have different functions in the hippocampal opioid system.

\subsection{Functional considerations}

Stress and steroid hormones both influence the hippocampal opioid system, and may contribute to sex differences in drug related learning. Projections from the CA2 region to the ventral tegmental area via the lateral septum are involved in relaying contextual information to the midbrain, and for example, are required for contextual reinstatement of cocaine seeking (Luo et al., 2011). Thus, our findings that pDOR-ir is prominent in the CA2 region suggests that activation of DORs can impact context-dependent motivational behaviors.

CA2 pyramidal neurons are strikingly less plastic than the other CA regions (Zhao et al., 2007). CA2 is protected from seizure-induced damage, yet experiences a greater loss of interneurons in schizophrenic brains compared to other hippocampal subregions (Mercer et al., 2007), which also highlights its functional distinction. Regarding the stress effects in estrus females, it is possible that glucocorticoids or other mediators that are elevated during acute stress overcome this functional rigidity allowing greater DOR activation in females, leading to increased neuronal activation in specific hippocampal regions. Moreover, our findings suggest that acute stress in females can impact DOR functions in these neurons, thereby modulating cell excitability, longterm potentiation and regulation of other signaling cascades.

Activation of DORs may directly and indirectly interact with the hippocampal brain-derived neurotrophic factor (BDNF) system. BDNF and its activated trkB receptor are contained in mossy fiber boutons, whereas BDNF mRNA and full-length trkB receptor are expressed in pyramidal cells [reviewed in Gray et al. (2012)]. Like the opioid system, the BDNF system is important for regulating synaptic plasticity (Schjetnan and Escobar, 2012) and is sensitive to both stress and ovarian hormone levels (Gray et al., 2012). DOR agonists have been shown to increase BDNF mRNA expression in the hippocampus (Torregrossa et al., 2006; Zhang et al., 2006; Torregrossa et al., 2004). This suggests that stress and/or gonadal 
hormones may affect the interplay between the DOR and BDNF systems in the hippocampus, but this remains to be further examined.

In conclusion, we have demonstrated the localization of the phosphorylated delta opioid receptor in the hippocampus, with high expression in the CA2 region specifically. In addition, estrous cycle phase impacts the expression of pDOR-ir in females; an effect which is dampened following acute immobilization stress. Together, this data supports recent literature that gonadal hormones and stress interact to modulate the excitability and plasticity of the hippocampus.

\section{Experimental procedure}

\subsection{Animals}

4.1.1. Cohorts-All procedures were approved by the Weill Cornell Medical College and Rockefeller University Institutional Animal Care and Use Committees and were in accordance with the 2011 Eighth Edition of the National Institutes of Health Guide for the Care and Use of Laboratory Animals. Adult male and female Sprague Dawley rats (2-3 months old, $N=76$ ) from Charles River Laboratories (Wilmington, MA) were housed 3-4 animals per cage with a 12:12 light/dark cycle and free access food and water. Three cohorts were housed in different animal facilities (WCMC and RU), and their brains were collected in different years. The first cohort, also used in our previous study (Lane et al., 2008), was used to assess if pDOR levels are altered after acute morphine exposure. The second cohort that was used to assess the effects of AIS in males and in females from different estrous stages, was the same used in our recent study (Gonzales et al., 2011). The third cohort, used in our previous studies (Torres-Reveron et al., 2008, 2009a, 2009b), was used to assess pDOR-ir in ovariectomized rats treated with estradiol benzoate. All animals were sacrificed between 9AM and $1 \mathrm{PM}$.

4.1.2. Estrous cycle determination-Only female rats that showed two consecutive, regular 4-5 day estrous cycles were used in these studies. Estrous stage was determined using vaginal smear cytology (Turner and Bagnara, 1971) daily between 9:00 and 10:00 AM, after one week of acclimation following arrival. To control for the effects of handling, male rats were removed from their cages daily to perform mock estrous cycling. Uterine weight and plasma serum estradiol levels via radioimmunoassay (Torres-Reveron et al., 2008) were measured to confirm estrous stage. Diestrus II rats rather than metestrus (diestrus I) were chosen to insure that rats were completely out of the estrus phase.

4.1.3.Ovariectomy and estradiol replacement-Rats were anesthetized with isofluorane (2-3\% in oxygen) and the ovaries were removed bilaterally as previously described (Torres-Reveron et al., 2009a). Ovariectomized rats received a single subcutaneous injection of $10 \mu \mathrm{g} / 0.2 \mathrm{ml}$ of estradiol benzoate (Sigma-Aldrich, St. Louis, MO) in sesame oil and were sacrificed $24 \mathrm{~h}$ later. Control rats were injected with sesame oil only.

4.1.4.Acute morphine administration-Male rats $(N=8)$ were injected with morphine $(20 \mathrm{mg} / \mathrm{kg}$, I.P.) or saline and sacrificed $1 \mathrm{~h}$ later. Since injected morphine sulfate has a halflife of 2-4 h, morphine was physiologically active in the animals' systems at the time they were sacrificed (Lane et al. 2008).

4.1.5.Acute immobilization stress-Rats $(N=48)$ were transported from their home room into a procedure room, and AIS was performed as previously described (Lucas et al., 2007; Shansky et al., 2010) between 9:00 AM and 1:00 PM. Rats were placed in plastic cone 
shaped polyethylene bags containing a small hole at the apex of the cone; a Kotex mini-pad was placed underneath them to collect urine. Rats were placed with their nose at the hole, sealed with tape in the bag, and left undisturbed on a countertop for $30 \mathrm{~min}$. Immediately after AIS, rats were anesthetized in the procedure room and their brains perfusion-fixed (see below). Control rats were left in the home-room and anesthetized prior to transfer to the procedure room for perfusion.

\subsection{Immunocytochemistry}

4.2.1. Antibodies-A rabbit polyclonal antibody generated against a synthetic phosphopeptide corresponding to residues surrounding Ser363 of human pDOR (\#3641 Cell Signaling, Danvers, MA) was used in this study. This antibody has been used to measure pDOR-ir using immunofluorescence, western blots and immunoprecipitation in both HEK293 cells and neuronal cells. Previously, in Western blots using this antibody on mouse hippocampal samples, a $65 \mathrm{kDa}$ band was seen in mice treated with DOR agonist SNC80 $(10 \mathrm{mg} / \mathrm{kg})$ and sacrificed $20 \mathrm{~min}$ later (Pradhan et al., 2009). This same band was not found in DOR knockout mice with the same treatment (Pradhan et al., 2009), further demonstrating specificity of this antibody. Although the manufacturer's supply of this antibody has been exhausted, reproduction of the antibody is expected in mid-2013 (personal communication with Cell Signaling). Serial dilution tests were used to show that the labeling intensity of the pDOR antibody was linear, and a dilution that produced slightly less than half-maximal labeling intensity was chosen for our studies to optimize the detection of intensity variations (Chang et al., 2000).

4.2.2. Section preparation-Rats were deeply anesthetized with sodium pentobarbital $(150 \mathrm{mg} / \mathrm{kg}$, I.P.) and perfused sequentially through the ascending aorta with: (1) $10-15 \mathrm{ml}$ saline containing $2 \%$ heparin in $0.9 \%$ saline; (2) $50 \mathrm{ml} 3.75 \%$ acrolein and $2 \%$ paraformaldehyde in 0.1 M phosphate buffer (PB; pH7.4); and (3) $200 \mathrm{ml} \mathrm{2 \%}$ paraformaldehyde in PB. After perfusion, brains were cut into $5 \mathrm{~mm}$ coronal blocks, postfixed in 2\% PFA in PB for $30 \mathrm{~min}$, and switched into PB. Coronal sections (40 mm thick) through the hippocampus were cut on a vibratome in PB. Sections were stored in cryoprotectant solution ( $30 \%$ sucrose and $30 \%$ ethylene glycol in PB) at $-20{ }^{\circ} \mathrm{C}$ (Milner et al., 2011b). For immunocytochemistry, sections were rinsed in PB, coded with hole-punches and pooled into containers to ensure identical processing (Pierce et al., 1999). Sections were incubated in $1 \%$ sodium borohydride in PB for 30 min and rinsed in PB.

\subsubsection{Light microscopic immunocytochemistry and analysis-To examine} changes in pDOR-ir levels between different experimental groups, sections were processed as previously described (Torres-Reveron et al., 2008). Briefly, sections were transferred to $0.1 \mathrm{M}$ Tris-buffered saline (TS; pH7.6) and then blocked in 0.5\% BSA in TS for $30 \mathrm{~min}$. Sections were placed in the pDOR antibody (1:500) for $24 \mathrm{~h}$ at room temperature, andthen $24-72 \mathrm{~h}$ at $4{ }^{\circ} \mathrm{C}$. Sections then were processed in a 1:400 dilution of biotinylated goat-anti rabbit immunoglobulin (IgG;Vector Laboratories, Burlingame, CA) for 30 min followed by a 1:100 dilution of avidin-biotin complex (ABC; Vectastain elite kit, Vector Laboratories) for $30 \mathrm{~min}$. All incubations were separated by washes of TS. Sections were reacted in 3,3'diaminobenzidine (DAB; Sigma-Aldrich, St. Louis, MO) and $3 \% \mathrm{H}_{2} \mathrm{O}_{2}$ in TS for 4-6 min and rinsed in TS followed by PB.Sections were mounted on gelatin-coated slides, dehydrated and coverslipped from xylene with DPX mounting media (Sigma-Aldrich).

Sections from the dorsal hippocampus ( -3.6 to $-4.0 \mathrm{~mm}$ from Bregma; Swanson 2000) were chosen for analysis. Cell layers were identified using terminology from the Swanson atlas (Swanson, 2000). An experimenter who was blind to treatment group did all evaluations. For quantitative densitometry, photomicrographs through regions of interest (ROI) were 
captured using a Dage MTI CCD-72 camera on a Nikon Eclipse 80i microscope and converted to gray-scale. The mean gray value (of $256 \mathrm{~Gy}$ levels) for each selected ROI was determined as previously described (Torres-Reveron et al., 2008). To control for variations in illumination and background staining, the average pixel density from three regions lacking labeling was subtracted. Optical density values were measured using Image J64 and net optical density values obtained after subtracting background values. All data was analyzed using a Two-Way ANOVA followed by Tukey HSD post-hoc analysis $(p<0.05)$.

\subsection{Electron microscopic immunocytochemistry and analysis}

4.3.1. Immunoperoxidase labeling-Sections were processed for light microscopy as described above and rinsed in PB. They then were placed into $2 \%$ osmium tetroxide in PB for $1 \mathrm{~h}$, then dehydrated in increasing concentrations of ethanol and propylene oxide before being embedded in Embed 812 (Electron Microscopy Science, Fort Washington, PA) (Milner et al., 2011b). Ultrathin sections (70-72 nm thick) through the ROI were cut on a Leica UCT ultratome and collected on 400-mesh copper grids (EMS). The grids were counterstained with uranyl acetate and Reynolds lead citrate. Sections were examined on a Tecnai Biotwin transmission electron microscope. Immunolabeled profiles were classified using defined morphological criteria (Peters et al., 1991). Dendritic profiles contained regular microtubule arrays and were usually postsynaptic to axon terminal profiles. Axon terminal profiles had numerous small synaptic vesicles.

For figures, images were adjusted for sharpness in Adobe Photoshop 9.0 and imported into PowerPoint 2010 on a Mac-Book where final adjustments to brightness and contrast were made. These changes did not alter the original content of the raw image. Graphs were generated in Prism 5.0a.

\section{Acknowledgments}

We thank Ms. Andreina Gonzalez for technical assistance.

\section{References}

Adams MM, Oung T, Morrison JH, Gore AC, et al. Length of postovariectomy interval and age, but not estrogen replacement, regulate $\mathrm{N}$-methyl-D-aspartate receptor mRNA levels in the hippocampus of female rats. Exp Neurol. 2001; 170:345-356. [PubMed: 11476600]

Amaral, D.; Lavenex, P., et al. Hippocampal neuroanatomy. In: Andersen, P.; Morris, R.; Amaral, D.; Bliss, T.; O'Keefe, J., editors. The Hippocampus Book. Oxford University Press; New York: 2007. p. 37-131.

Chang PC, Aicher SA, Drake CT, et al. Kappa opioid receptors in rat spinal cord vary across the estrous cycle. Brain Res. 2000; 861:168-172. [PubMed: 10751578]

Cheng Y, Tao YM, Sun JF, Wang YH, Xu XJ, Chen J, Chi ZQ, Liu JG, et al. Adenosine A(1) receptor agonist N(6)-cyclohexyl-adenosine induced phosphorylation of delta opioid receptor and desensitization of its signaling. Acta Pharmacol Sin. 2010; 31:784-790. [PubMed: 20562901]

Cohen GA, Doze VA, Madison DV, et al. Opioid inhibition of GABA release from presynaptic terminals of rat hippocampal interneurons. Neuron. 1992; 9:325-335. [PubMed: 1497896]

Commons KG, Milner TA, et al. Ultrastructural heterogeneity of enkephalin-containing neurons in the rat hippocampal formation. J Comp Neurol. 1995a; 358:324-342. [PubMed: 7560290]

Commons KG, Milner TA, et al. Localization of delta opiate receptor immunoreactivity in interneurons and pyramidal cells in the rat hippocampus. J Comp Neurol. 1997; 381:373-387. [PubMed: 9133574]

Conrad CD, Grote KA, Hobbs RJ, Ferayorni A, et al. Sex differences in spatial and non-spatial Ymaze performance after chronic stress. Neurobiol Learn Mem. 2003; 79:32-40. [PubMed: 12482677] 
Czeh B, Simon M, van der Hart MG, Schmelting B, Hesselink MB, Fuchs E, et al. Chronic stress decreases the number of parvalbumin-immunoreactive interneurons in the hippocampus: prevention by treatment with a substance P receptor (NK1) antagonist. Neuropsychopharmacology. 2005; 30:67-79. [PubMed: 15470372]

Drake CT, Chavkin C, Milner TA, et al. Opioid systems in the dentate gyrus. Prog Brain Res. 2007; 163:245-814. [PubMed: 17765723]

Elman I, Karlsgodt KH, Gastfriend DR, et al. Gender differences in cocaine craving among nontreatment-seeking individuals with cocaine dependence. Am J Drug Alcohol Abuse. 2001; 27:193-202. [PubMed: 11417935]

Galea LAM, McEwen BS, Tanapat P, Deak T, Spencer RL, Dhabhar FS, et al. Sex differences in dendritic atrophy of CA3 pyramidal neurons in response to chronic restraint stress. Neuroscience. 1997; 81:689-697. [PubMed: 9316021]

Gonzales KL, Chapleau JD, Pierce JP, Kelter DT, Williams TJ, Torres-Reveron A, McEwen BS, Waters EM, Milner TA, et al. The influences of reproductive status and acute stress on the levels of phosphorylated mu opioid recetpor immunoreactivity in rat hippocampus. Front Neuroendocrinol. 2011; 2:1-10.

Gray JD, Milner TA, McEwen BS, et al. Dynamic plasticity: the role of glucocorticoids, brain-derived neurotrophic factor and other trophic factors. Neuroscience. 2012:10.

Grove KL, Campbell RE, Ffrench-Mullen JM, Cowley MA, Smith MS, et al. Neuropeptide Y Y5 receptor protein in the cortical/limbic system and brainstem of the rat: expression on gammaaminobutyric acid and corticotropin-releasing hormone neurons. Neuroscience. 2000; 100:731740. [PubMed: 11036207]

Hu W, Zhang M, Czeh B, Flugge G, Zhang W, et al. Stress impairs GABAergic network function in the hippocampus by activating nongenomic glucocorticoid receptors and affecting the integrity of the parvalbumin-expressing neuronal network. Neuropsychopharmacology. 2010; 35:1693-1707. [PubMed: 20357756]

Hyman SE, Malenka RC, et al. Addiction and the brain: the neurobiology of compulsion and its persistence. Nat Rev Neurosci. 2001; 2:695-703. [PubMed: 11584307]

Jones MW, McHugh TJ, et al. Updating hippocampal representations: CA2 joins the circuit. Trends Neurosci. 2011; 34:526-535. [PubMed: 21880379]

Khalid, S.; Chapleau, JD.; Williams, TJ.; Schierberl, K.; Guarjardo, HM.; Waters, EM.; Milner, TA. Acute immoblization stress alters the subcellular distribution of mu opioid receptor immunoreactivity in hippocampal parvalbumin-containg interneurons of female rats, Program No 186.11; Neuroscience Meeting Planner; Washington DC. Society for Neuroscience, 2010; 2010. Online

Kitraki E, Kremmyda O, Youlatos D, Alexis MN, Kittas C, et al. Gender-dependent alterations in corticosteroid receptor status and spatial performance following 21 days of restraint stress. Neuroscience. 2004; 125:47-55. [PubMed: 15051144]

Koch T, Hollt V, et al. Role of receptor internalization in opioid tolerance and dependence. Pharmacol Ther. 2008; 117:199-206. [PubMed: 18076994]

Koob GF, et al. Neurobiological substrates for the dark side of compulsivity in addiction. Neuropharmacology. 2008

Koob GF, Sanna PP, Bloom FE, et al. Neuroscience of addiction. Neuron. 1998; 21:467-476. [PubMed: 9768834]

Lane DA, Lessard AA, Chan J, Colago EE, Zhou Y, Schlussman SD, Kreek MJ, Pickel VM, et al. Region-specific changes in the subcellular distribution of AMPA receptor GluR1 subunit in the rat ventral tegmental area after acute or chronic morphine administration. J Neurosci. 2008; 28:96709681. [PubMed: 18815253]

Lucas LR, Wang CJ, McCall TJ, McEwen BS, et al. Effects of immobilization stress on neurochemical markers in the motivational system of the male rat. Brain Res. 2007; 1155:108-115. [PubMed: 17511973]

Luine VN, Beck KD, Bowman RE, Frankfurt M, MacLusky NJ, et al. Chronic stress and neural function: accounting for sex and age 19, 743-751. J Neuroendocrinol. 2007:19, 743-751. 
Luo AH, Tahsili-Fahadan P, Wise RA, Lupica CR, AstonJones G, et al. Linking context with reward: a functional circuit from hippocampal CA3 to ventral tegmental area. Science. 2011; 333:353-357. [PubMed: 21764750]

Magarinos AM, Verdugo JM, McEwen BS, et al. Chronic stress alters synaptic terminal structure in hippocampus. Proc Natl Acad Sci USA. 1997; 94:14002-14008. [PubMed: 9391142]

Magloczky Z, Acsady L, Freund TF, et al. Principal cells are the postsynaptic targets of supramammillary afferents in the hippocampus of the rat. Hippocampus. 1994; 4:322-334. [PubMed: 7531093]

McEwen BS, et al. Stress and hippocampal plasticity. Annu Rev Neurosci. 1999; 22:105-122. [PubMed: 10202533]

McEwen BS, Milner TA, et al. Hippocampal formation: shedding light on the influence of sex and stress on the brain. Brain Res Rev. 2007; 55:343-355. [PubMed: 17395265]

McKay JR, Rutherford MJ, Cacciola JS, Kabasakalian-McKay R, Alterman AI, et al. Gender differences in the relapse experiences of cocaine patients. J Nerv Ment Dis. 1996; 184:616-622. [PubMed: 8917159]

Mercer A, Trigg HL, Thomson AM, et al. Characterization of neurons in the CA2 subfield of the adult rat hippocampus. J Neurosci. 2007; 27:7329-7338. [PubMed: 17611285]

Milner, TA.; Marrone, GF.; Williams, TJ.; Schierberl, KC.; Waters, EM.; McEwen, BS.; Gonzales, KL. Chronic stress alters mu opioid receptor trafficking in parvalbumin-containing neurons in the female rat hippocampus Program No 325.07; Neuroscience Meeting Planner; Washington DC. Society for Neuroscience, 2011; 2011a. Online

Milner, TA.; Waters, EM.; Robinson, DC.; Pierce, JP., et al. Degenerating processes identified by electron microscpic immunocytochemical methods. In: Manfredi, G.; Kawamata, H., editors. Neurodegeneration, Methods and Protocols. Springer; New York: 2011b. p. 23-59.

Nestler EJ, et al. From neurobiology to treatment: progress against addiction. Nat Neurosci. 2002; 5(Suppl. 1076-1079):1076-1079. [PubMed: 12403990]

Ochiishi T, Saitoh Y, Yukawa A, Saji M, Ren Y, Shirao T, Miyamoto H, Nakata H, Sekino Y, et al. High level of adenosine A1 receptor-like immunoreactivity in the CA2/CA3a region of the adult rat hippocampus. Neuroscience. 1999; 93:955-967. [PubMed: 10473260]

Peters, A.; Palay, SL.; Webster, H deF, et al. The Fine Structure of the Nervous System. 3rd. Oxford University Press; New York: 1991.

Pierce JP, Kurucz O, Milner TA, et al. The morphometry of a peptidergic transmitter system before and after seizure. I. Dynorphin B-like immunoreactivity in the hippocampal mossy fiber system. Hippocampus. 1999; 9:255-276. [PubMed: 10401641]

Pradhan AA, Becker JA, Scherrer G, Tryoen-Toth P, Filliol D, Matifas A, Massotte D, Gaveriaux-Ruff C, Kieffer BL, et al. In vivo delta opioid receptor internalization controls behavioral effects of agonists. PLoS One. 2009; 4:e5425. [PubMed: 19412545]

Quock RM, Burkey TH, Varga E, Hosohata Y, Hosohata K, Cowell SM, Slate CA, Ehlert FJ, Roeske WR, Yamamura HI, et al. The delta-opioid receptor: molecular pharmacology, signal transduction, and the determination of drug efficacy. Pharmacol Rev. 1999; 51:503-532. [PubMed: 10471416]

Redila VA, Chavkin C, et al. Stress-induced reinstatement of cocaine seeking is mediated by the kappa opioid system. Psychopharmacology (Berl). 2008; 200:59-70. [PubMed: 18575850]

Rubin A, Stout RL, Longabaugh R, et al. Gender differences in relapse situations. Addiction. 1996; 91(Suppl. S111- 120):S111-S120. [PubMed: 8997785]

Scherrer G, Tryoen-Toth P, Filliol D, Matifas A, Laustriat D, Cao YQ, Basbaum AI, Dierich A, Vonesh JL, GaveriauxRuff C, Kieffer BL, et al. Knockin mice expressing fluorescent delta-opioid receptors uncover G protein-coupled receptor dynamics in vivo. Proc Natl Acad Sci USA. 2006; 103:9691-9696. [PubMed: 16766653]

Schjetnan AG, Escobar ML, et al. In vivo BDNF modulation of hippocampal mossy fiber plasticity induced by high frequency stimulation. Hippocampus. 2012; 22:1-8. [PubMed: 20848610]

Shansky RM, Hamo C, Hof PR, Lou W, McEwen BS, Morrison JH, et al. Estrogen promotes stress sensitivity in a prefrontal cortex-amygdala pathway. Cereb Cortex. 2010; 20:2560-2567. [PubMed: 20139149] 
Simmons ML, Chavkin C, et al. Endogenous opioid regulation of hippocampal function. Int Rev Neurobiol. 1996; 39(145-96):145-196. [PubMed: 8894847]

Simons SB, Caruana DA, Zhao M, Dudek SM, et al. Caffeine-induced synaptic potentiation in hippocampal CA2 neurons. Nat Neurosci. 2011; 15:23-25. [PubMed: 22101644]

Stumm RK, Zhou C, Schulz S, Hollt V, et al. Neuronal types expressing mu- and delta-opioid receptor mRNA in the rat hippocampal formation. J Comp Neurol. 2004; 469:107-118. [PubMed: 14689476]

Tanapat P, Hastings NB, Gould E, et al. Ovarian steroids influence cell proliferation in the dentate gyrus of the adult female rat in a dose- and time-dependent manner. J Comp Neurol. 2005; 481:252-265. [PubMed: 15593136]

Torregrossa MM, Isgor C, Folk JE, Rice KC, Watson SJ, Woods JH, et al. The delta-opioid receptor agonist (+) BW373U86 regulates BDNF mRNA expression in rats. Neuropsychopharmacology. 2004; 29:649-659. [PubMed: 14647482]

Torregrossa MM, Jutkiewicz EM, Mosberg HI, Balboni G, Watson SJ, Woods JH, et al. Peptidic delta opioid receptor agonists produce antidepressant-like effects in the forced swim test and regulate BDNF mRNA expression in rats. Brain Res. 2006; 1069:172-181. [PubMed: 16364263]

Torres-Reveron A, Khalid S, Williams TJ, Waters EM, Drake CT, McEwen BS, Milner TA, et al. Ovarian steroids modulate leu-enkephalin levels and target leu-enkephalinergic profiles in the female hippocampal mossy fiber pathway. Brain Res. 2008; 1232(70-84):70-84. [PubMed: 18691558]

Torres-Reveron A, Khalid S, Williams TJ, Waters EM, Jacome L, Luine VN, Drake CT, McEwen BS, Milner TA, et al. Hippocampal dynorphin immunoreactivity increases in response to gonadal steroids and is positioned for direct modulation by ovarian steroid receptors. Neuroscience. 2009a; 159:204-216. [PubMed: 19150393]

Torres-Reveron A, Williams TJ, Chapleau JD, Waters EM, McEwen BS, Drake CT, Milner TA, et al. Ovarian steroids alter mu opioid receptor trafficking in hippocampal parvalbumin GABAergic interneurons. Exp Neurol. 2009b; 219:319-327. [PubMed: 19505458]

Turner, CD.; Bagnara, JT., et al. General Endocrinology. W. B. Saunders; Philadelphia: 1971.

Vertes RP, McKenna JT, et al. Collateral projections from the supramammillary nucleus to the medial septum and hippocampus. Synapse. 2000; 38:281-293. [PubMed: 11020231]

Vyas A, Mitra R, Shankaranarayana Rao BS, Chattarji S, et al. Chronic stress induces contrasting patterns of dendritic remodeling in hippocampal and amygdaloid neurons. J Neurosci. 2002; 22:6810-6818. [PubMed: 12151561]

Wang ZJ, Wang LX, et al. Phosphorylation: a molecular switch in opioid tolerance. Life Sci. 2006; 79:1681-1691. [PubMed: 16831450]

Williams TJ, Akama KT, Knudsen MG, McEwen BS, Milner TA, et al. Ovarian hormones influence corticotropin releasing factor receptor colocalization with delta opioid receptors in CA1 pyramidal cell dendrites. Exp Neurol. 2011a; 230:186-196. [PubMed: 21549703]

Williams TJ, Milner TA, et al. Delta opioid receptors colocalize with corticotropin releasing factor in hippocampal interneurons. Neuroscience. 2011; 179:9-22. [PubMed: 21277946]

Williams TJ, Torres-Reveron A, Chapleau JD, Milner TA, et al. Hormonal regulation of delta opioid receptor immunoreactivity in interneurons and pyramidal cells in the rat hippocampus. Neurobiol Learn Mem. 2011b; 95:206-220. [PubMed: 21224009]

Zhang H, Torregrossa MM, Jutkiewicz EM, Shi YG, Rice KC, Woods JH, Watson SJ, Ko MC, et al. Endogenous opioids upregulate brain-derived neurotrophic factor mRNA through delta- and micro-opioid receptors independent of antidepressant-like effects. Eur J Neurosci. 2006; 23:984994. [PubMed: 16519663]

Zhao M, Choi YS, Obrietan K, Dudek SM, et al. Synaptic plasticity (and the lack there of) in hippocampal CA2 neurons. J Neurosci. 2007; 27:12025-12032. [PubMed: 17978044] 

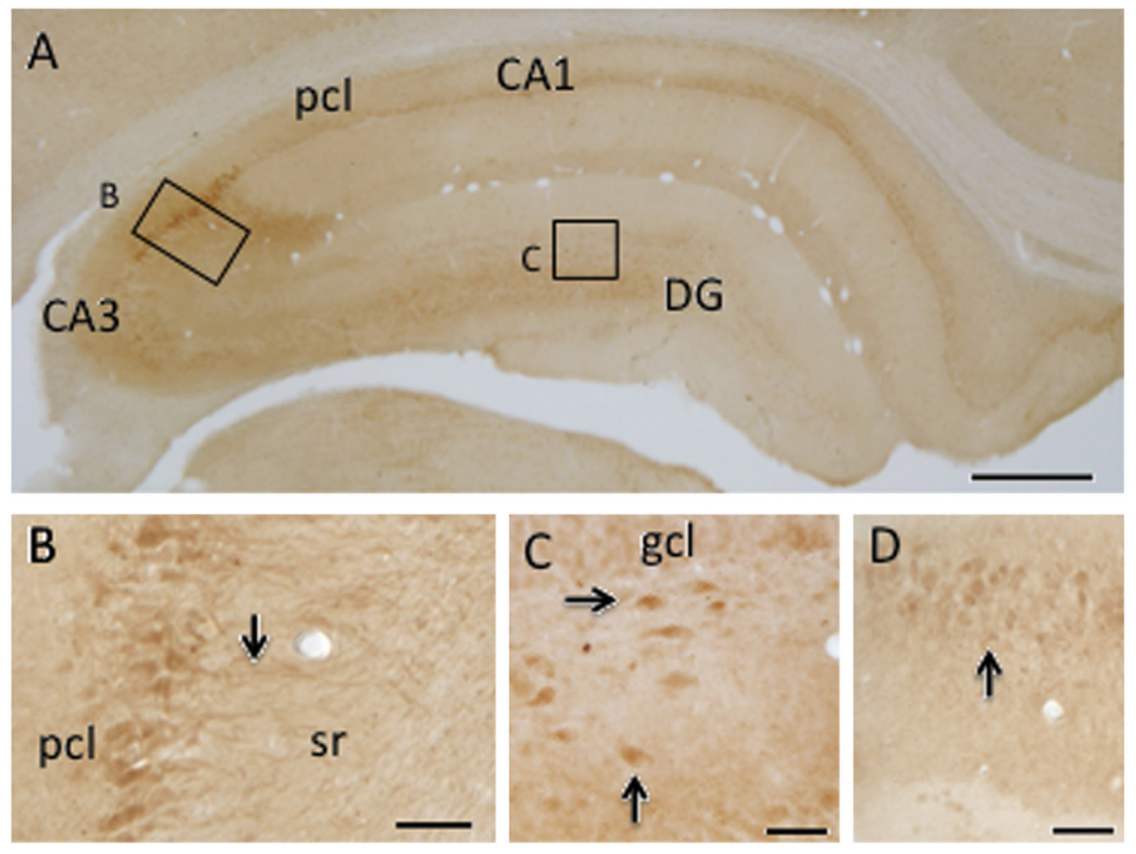

Fig. 1.

By light microscopy, pDOR is distributed in select subregions of the hippocampus. (A) pDOR-ir is most dense in the pyramidal cell layer ( $\mathrm{pcl}$ ), especially in CA2/CA3a (boxed region enlarged in B). Additionally, pDOR-labeled cells are found in the hilus of the dentate gyrus (DG; boxed region enlarged in C). (B) Higher magnification of the CA2/CA3a region shows dense pDOR-labeled somata in the pyramidal cell layer (pcl) and in dendrites (arrow) in stratum radiatum (sr). (C) Higher magnification shows pDOR-labeled interneurons (arrows) in the hilus. (D) pDOR-ir is found in somata in the pyramidal cell layer (arrow) of the fasciola cinera. gcl=granule cell layer. Scale bars $=a, 100 \mu \mathrm{m}$ and $b-d, 10 \mu \mathrm{m}$. 


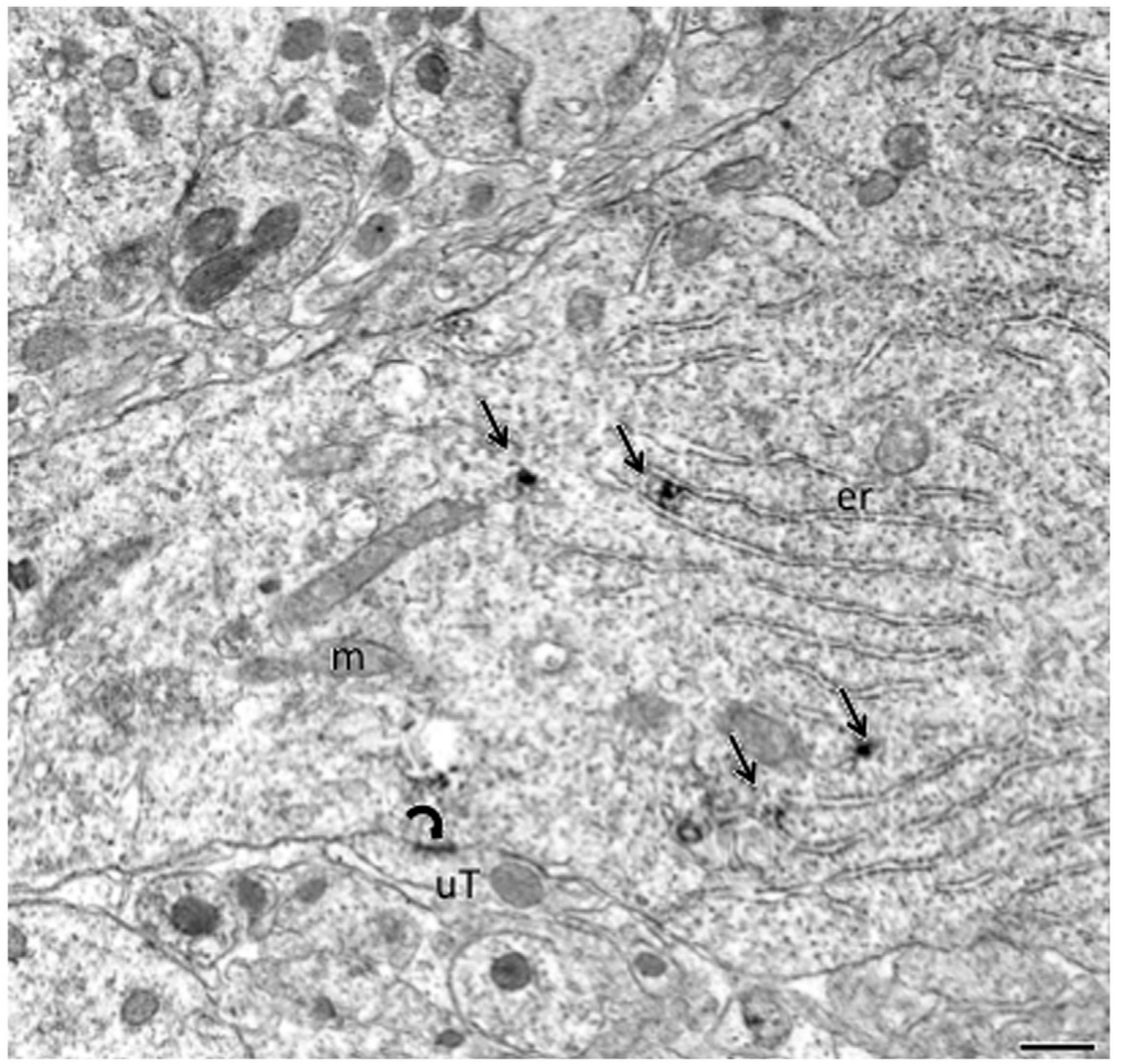

Fig. 2.

By electron microscopy, pDOR-ir is prominent in pyramidal cells in CA2/CA3a. Patches (arrows) of pDOR-ir are located near the endoplasmic reticulum (er) within the cytoplasm of proximal dendrites. An unlabeled terminal (uT) forms symmetric synapse (curved arrow) with the dendrite. $\mathrm{m}=$ mitochondria. Scale bar=500 $\mathrm{nm}$. 

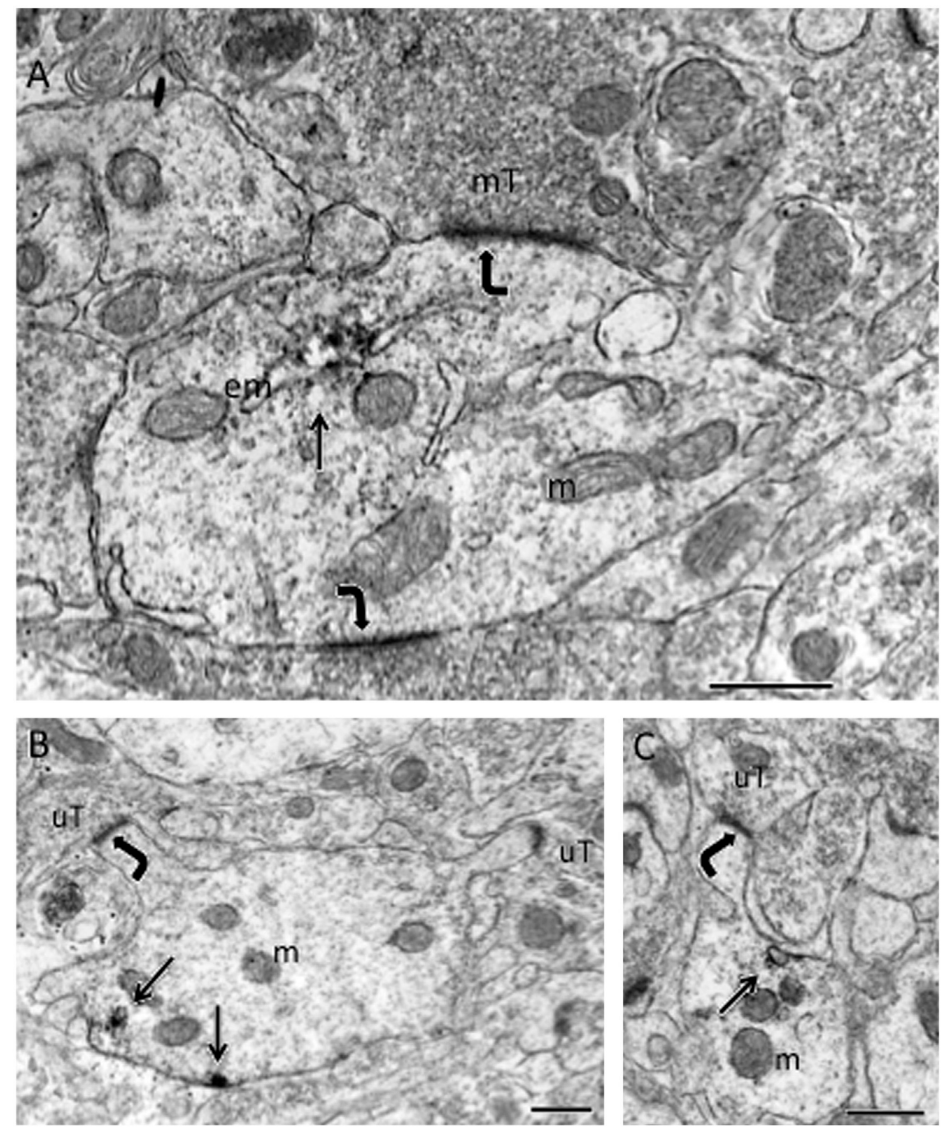

Fig. 3.

By electron microscopy, pDOR-ir is found in dendrites in CA2/3a. (A) pDOR-ir localized to endomembranes (em) in the shaft of a large dendrite, which is contacted (curved arrows) by mossy fiber terminals (mT). (B) Patches (arrows) of pDOR-ir are found near the plasma membrane of a dendrite. (C) A patch of pDOR-ir is adjacent to an endomembrane (arrow) near plasma membrane in a dendrite. In both $\mathrm{B}$ and $\mathrm{C}$, the spines contacted (curved arrows) by unlabeled terminals (uT) emanate from the shafts of the pDOR-labeled dendrite. Scale bars $=500 \mathrm{~nm}$. 

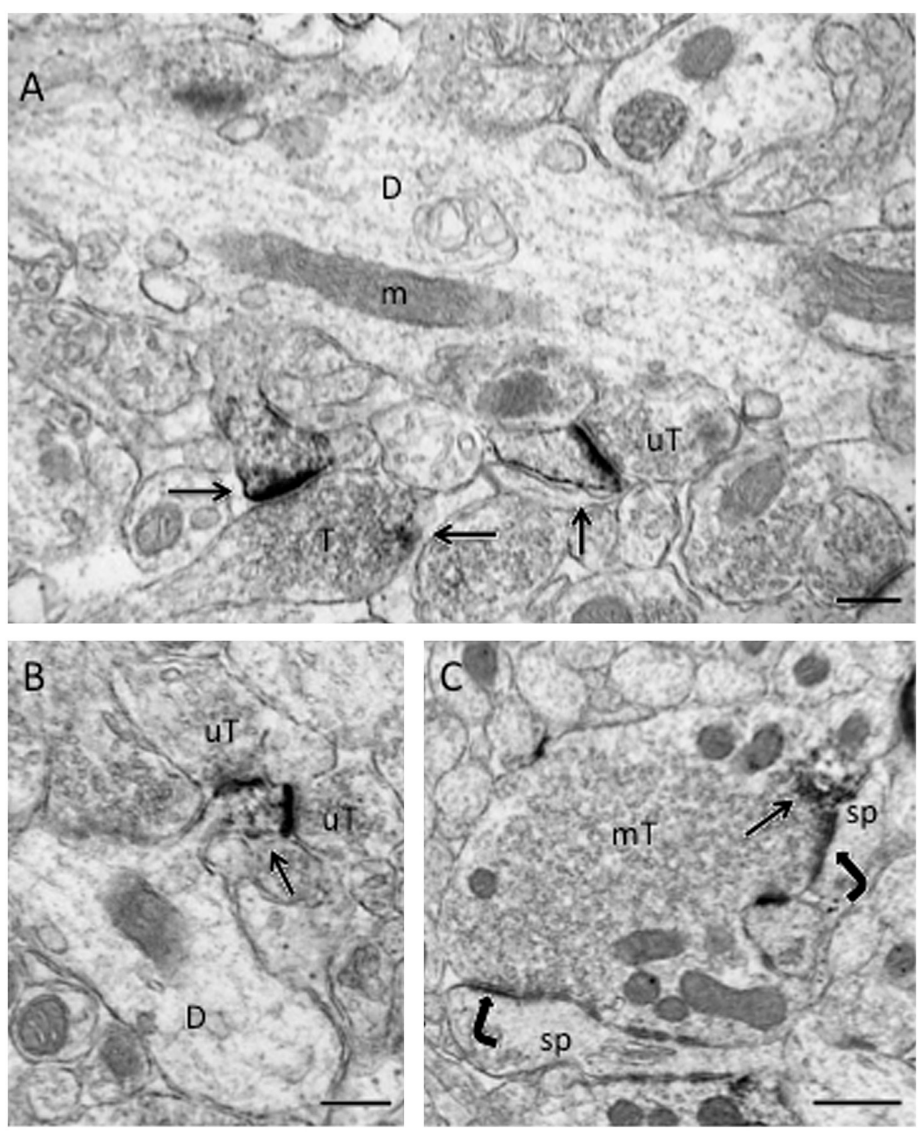

Fig. 4.

By electron microscopy, pDOR-ir is primarily in dendrites but also in terminals in the hilus of the dentate gyrus. (A) Dense pDOR-ir is found in the head of spines (arrows). The spine on the left emanates from a dendritic shaft (D) and is contacted by a small terminal (T), which contains a patch of pDOR-ir (arrow). The spine on the right is contacted by an unlabeled terminal (uT). (B) pDOR-ir (arrow) is in the head of a dendritic spine that emanates from a dendritic shaft (D) and is contacted by two unlabeled terminals (uT). C. pDOR-ir is affiliated with a cluster of vesicles (arrow) near the plasma membrane of a mossy fiber terminal (mT) that contacts (curved arrows) two dendritic spines (sp). Scale bars $=500 \mathrm{~nm}$. 
ㅁ Saline

- Acute Morphine

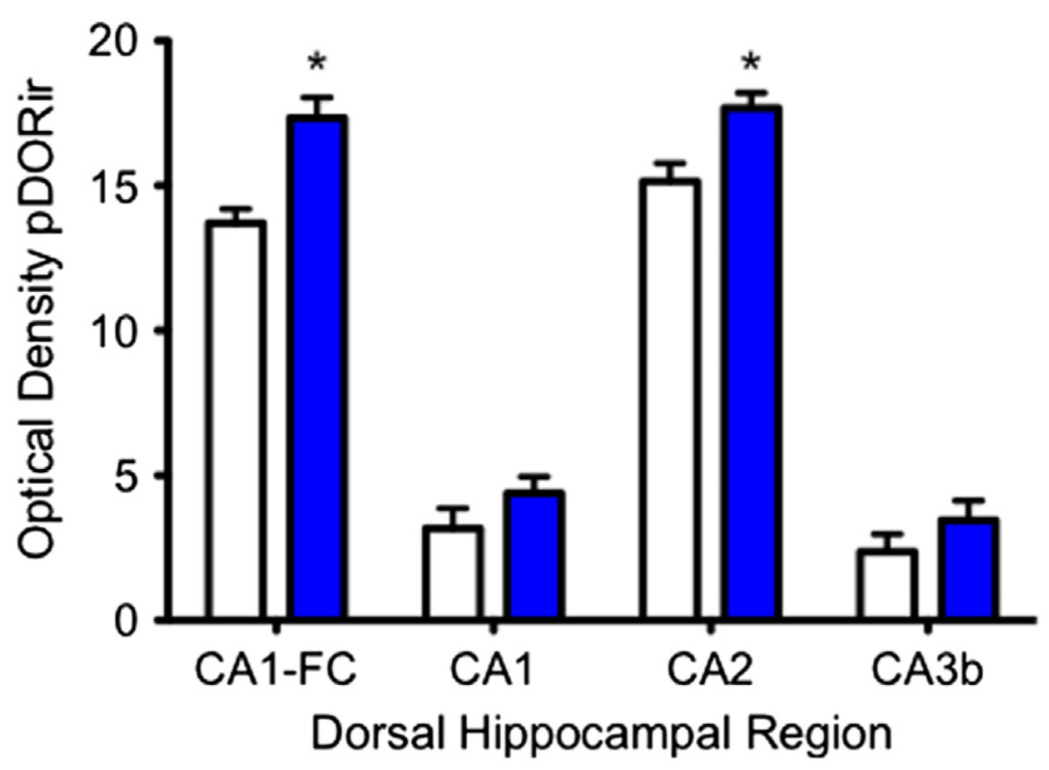

Fig. 5.

Acute morphine increases pDOR-ir in select hippocampal subregions. To determine an opioid agonist can increase pDOR-ir, male rats were injected with morphine. Using quantitative densitometry, pDOR-ir was examined in control animals and morphine treated animals in different hippocampal regions. Post-hoc analysis revealed that $\mathrm{pDOR}$-ir is significantly greater $(p=0.0001)$ in the $\mathrm{CA} 2 / 3 \mathrm{a}$ and in the CA1 - fasciola cinera $(\mathrm{FC})$ regions of male rats $1 \mathrm{~h}$ following a single morphine injection $(20 \mathrm{mg} / \mathrm{kg}$, I.P.). $N=4$ animals per condition. 


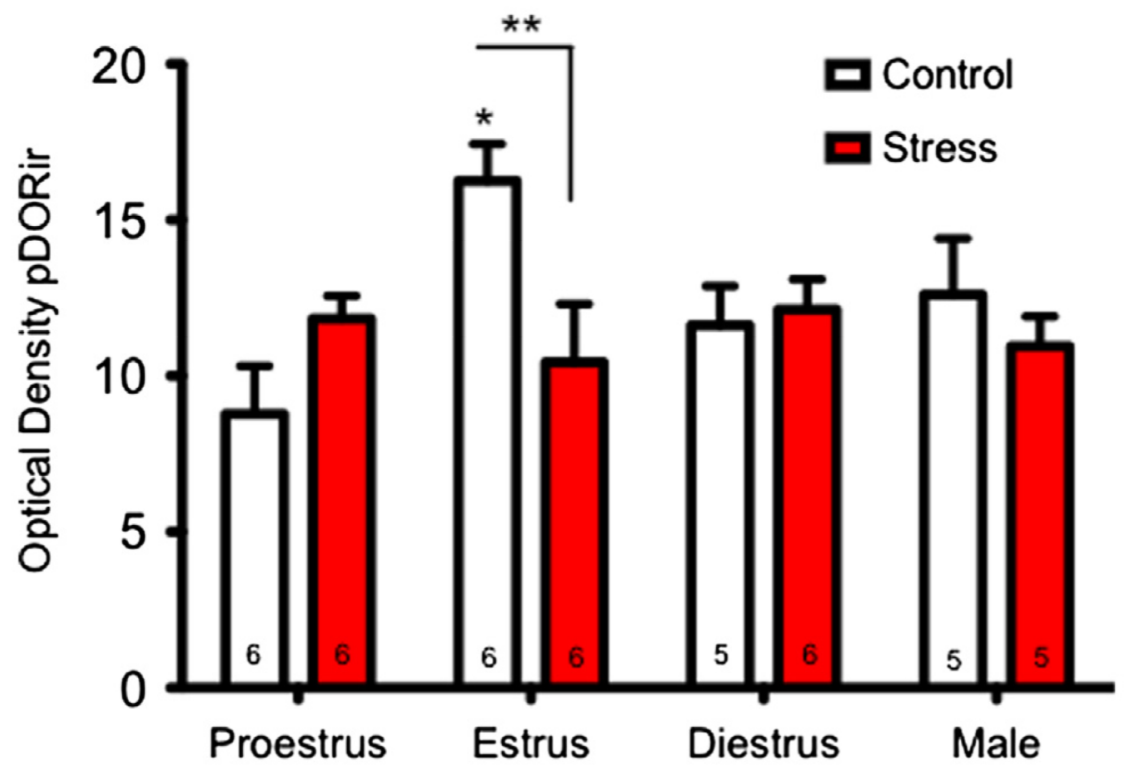

Fig. 6.

Effects of sex and estrous cycle stage on pDOR-ir in CA2/3a in normal and stressed rats. Quantitative densitometry was used to measure pDOR-ir in the CA2/CA3a region of the hippocampus in animals following acute immobilization stress and in control animals. Posthoc analysis revealed that in control animals, pDOR-ir is elevated in estrus compared to other cycle stages $(* p<0.05)$. Also, estrus animals were the only group showing a significant decrease $(* * p<0.05)$ in pDOR-ir following acute immobilization stress. 